American J. of Engineering and Applied Sciences 2 (4): 771-774, 2009

ISSN 1941-7020

(C) 2009 Science Publications

\title{
Wireless Phones Electromagnetic Field Radiation Exposure Assessment
}

\author{
${ }^{1}$ A.D. Usman, ${ }^{1}$ W.F. Wan Ahmad, ${ }^{1}$ M.Z.A. Ab Kadir and ${ }^{2}$ M. Mokhtar \\ ${ }^{1}$ Department of Electrical and Electronic Engineering, Faculty of Engineering, \\ University Putra Malaysia, 43400 UPM Serdang, Selangor, Malaysia \\ ${ }^{2}$ Department of Computer and Communication Engineering, Faculty of Engineering, \\ University Putra Malaysia, 43400 UPM Serdang, Selangor, Malaysia
}

\begin{abstract}
Problem statement: Inadequate knowledge of electromagnetic field emitted by mobile phones and increased usage at close proximity, created a lot of skepticism and speculations among end users on its safety or otherwise. Approach: In this study, near field electromagnetic field radiation measurements were conducted on different brand of mobile phones in active mode using a tri-axis isotropic probe and electric field meter. Results: The highest electromagnetic field exposure was recorded when the mobile phones are at outgoing call mode and backing the probe, which is higher in comparison to ICNIRP guidelines for exposure to general public. Conclusion: According to this finding, some mobile phones electromagnetic field radiation were found to be lower than the ICNIRP guidelines while some were far above the guidelines. Electromagnetic field intensity however, depends on the mode of operation and proximity of the mobile phones to the end user; hence it is safer to use mobile phones at SMS mode.
\end{abstract}

Key words: Electromagnetic field radiation, active mode, exposure level, wireless phones

\section{INTRODUCTION}

Since 1990 when cellular concept was first developed, the society has continued to witness the presence of electromagnetic fields in their environment arising from everyday applications. The sources of electromagnetic fields arise from power lines, mobile phones and broadcast antennas to mention just a few. To date there are about 4 billion individual subscribers worldwide accounting to $60 \%$ of the mobile penetration rate $^{[1]}$.

Mobile phones, found almost in every homes operate with low power, however, its closest proximity with human body and the human brains and body depending on electrical impulses to conduct complex life processes including the ability to read, recall and respond calls for more concern. Power transmitted from mobile phones during a call is dependent on the received coverage level from the base station. In cellular technology all transmitting devices, be it mobile phones or base stations, are designed to transmit the lowest amount of powers as required to sustain a call. The technology that achieves this communication between the mobile phone and the base station depend on the system which the mobile phone operators have adopted. Some current technologies include: Code Division Multiple Access (CDMA), Global System for
Mobile Communication (GSM) and Universal Mobile Telecommunication System (UMTS) for digital communications. Each network operator has a unique assigned radio frequency from defined standard frequencies allocated to each technology.

Worldwide, the mobile phone system is often referred to as 'cellular' telephone technology because the regions covered are broken into "cells" and each of these cells has their own localized service provided by a base station antenna. Currently, both the GSM and CDMA systems and WCDMA are used in mobile networks worldwide.

These technologies uses electromagnetic in RF ranges and the human body is particularly sensitive to RF EM fields. At these high frequencies the human body will absorb a significant amount of the radiated energy from these technologies ${ }^{[2-4]}$. This however, is causing an increasing public concern of possible health effects that may be associated with these exposures ${ }^{[5-7]}$.

\section{MATERIALS AND METHODS}

The EM field radiated from the sampled mobile phones of different brands and model were measured from a G-TEM cell and in an open space, in both a worst case was recorded.

Corresponding Author: A.D. Usman, Department of Electrical and Electronic Engineering, Faculty of Engineering, University Putra Malaysia, 43400 UPM Serdang, Selangor, Malaysia Tel: +60126627419 
An isotropic probe manufactured from Narda instruments was also used with PMM8053A electric field meter. The probes measures electric fields in $\mathrm{XYZ}$ planes and perform the vector addition of the individual readings and send the results on the display provided by the meter. The frequency range of the probe is $100 \mathrm{kHz}$ to $3 \mathrm{GHz}$ and electrical field strength of $10 \mathrm{mV} / \mathrm{m}-100 \mathrm{~V} / \mathrm{m}$, the room ambient field was recorded as $0.1 \mathrm{~V} / \mathrm{m}$ and was subtracted from the actual readings. Measurements were performed at close proximity less than $5 \mathrm{~cm}$ away from the probe for four different mobile phone modes, i.e., incoming call, outgoing call, receiving message and sending message modes.

The different types and models of the GSM phones under taste were fully charged and placed facing the field probe at similar position of the ear. Thereafter the position of the phones was then changed to measure the field strength at the back of the phone similar to how the phones back our body in the pocket. At various phases and positions only the maximum values recorded. The set up of such assessment is as shown in Fig. 1.

With the determination of the electric field strength, other parameters meant for the establishment of possible health risk associated with EM exposure can be obtained by incident field dosimetry and external field dosimetry. Various techniques for determination of internal field at near field in the body tissue can be found in $^{[8-10]}$. The heating effect is directly related to the total power absorbed by human body. Calculation procedure can be found based on Boundary Element Method $(\mathrm{BEM})$ as reported in $^{[10,11]}$ using Pocklinton equation.

At GSM frequency near $900 \mathrm{MHz}$, the average conductivity of a body $\delta=1.4 \mathrm{~S} \mathrm{~m}^{-1}$ and the corresponding average permittivity is $\epsilon=55$. Therefore, Eq. 1-5 show the relationship of the electric field, the dissipated power density as well as Specific Absorption Rate (SAR) which can be evaluated from the measurement results shown in Fig. 2-4. Also, in the medium with finite conductivity $\delta$, the dissipated power density $P_{d}$ can be obtained using Eq. 3 and 4 where $J$ is the current density, $\mathrm{C}$ is the specific heat capacity, $\mathrm{T}$ is the temperature and $\rho$ is the tissue density:

$$
\begin{aligned}
& E=\frac{J}{\delta+j \omega \varepsilon} \\
& \text { SAR }=\frac{d}{d t} \frac{d w}{d m}=\frac{d}{d t} \frac{d w}{\rho d v} \\
& \text { SAR }=C \frac{d T}{d t}
\end{aligned}
$$

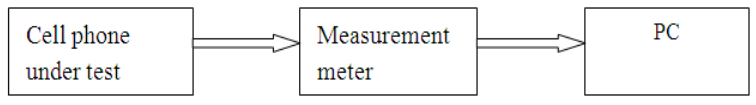

Fig. 1: Wireless phones EMF radiation exposure assessment set up

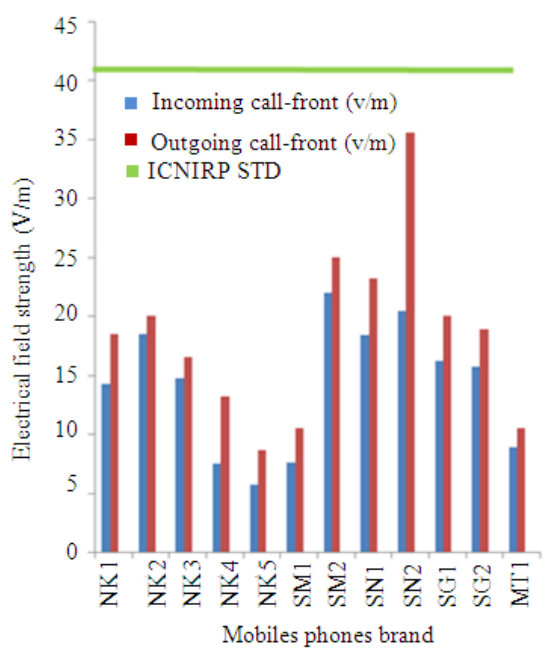

Fig. 2: Maximum electric field strength in the vicinity of the mobile phones measured during the incoming and outgoing call mode with the Phone facing the probe

$\mathrm{Pd}=\delta \mathrm{IEI^{2 }}$

$\mathrm{SAR}=\frac{\mathrm{IJI}^{2}}{\delta \rho}=\frac{\mathrm{IEI}^{2}}{\delta \rho}=\frac{\mathrm{Pd}}{\delta \rho}$

\section{RESULTS}

Results are obtained in terms of highest readings of the electric field strength in the vicinity of the mobile phones during incoming and outgoing call modes and receiving and sending message modes. These results are plotted against the 12 brand mobile phones, shown in Fig. 2-4 and compared with the ICNIRP guidelines for exposure to general public.

From Fig. 2, it is shown that the field strength emitted by the phones during the incoming and outgoing call mode with the phones facing the probe are well below the ICNIRP guidelines for limiting exposure to general public of $41.25 \mathrm{~V} / \mathrm{m}$. The highest reading obtained with brand $\mathrm{SN} 2$ in the outgoing call mode is $35.6 \mathrm{~V} / \mathrm{m}$ and the lowest reading obtained with brand NK5 during the incoming call mode is $5.7 \mathrm{~V} / \mathrm{m}$. 


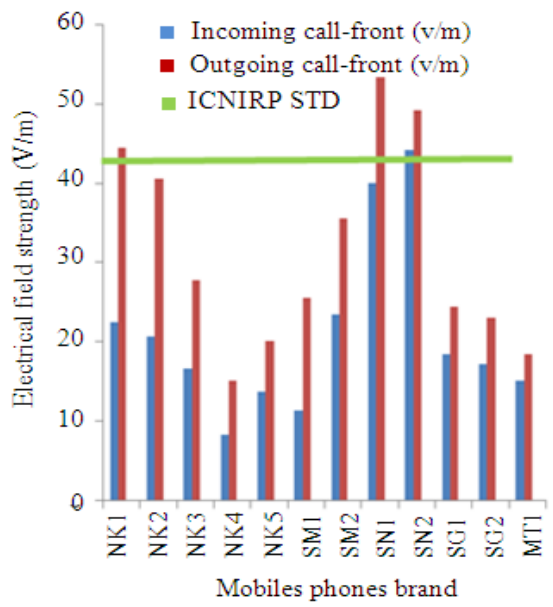

Fig. 3: Maximum electric field strength in the vicinity of the mobile phones measured during the incoming and outgoing call mode with the phone backing the probe

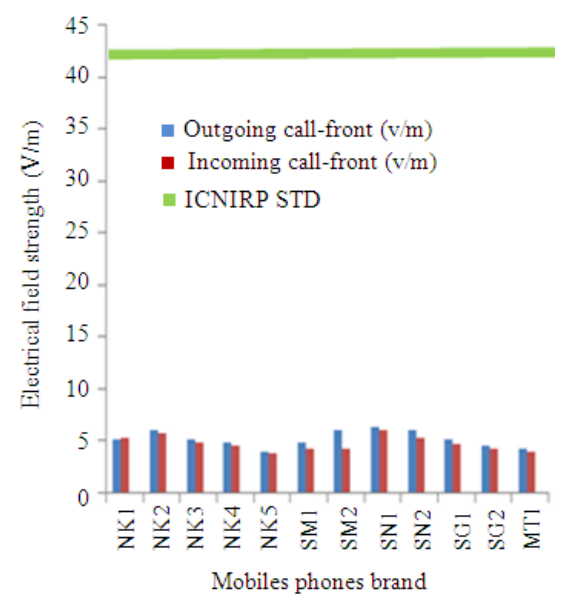

Fig. 4: Maximum electric field strength in the vicinity of the mobile phones measured during the receiving and sending message mode

In Fig. 3, maximum electric field strength in the vicinity of the mobile phones measured during the incoming and outgoing call mode with the phone backing the probe was shown. During this mode, the highest and lowest readings of the electric field strength are at 53.40 and $15.20 \mathrm{~V} / \mathrm{m}$, as demonstrated by brand SN1 and brand NK4 respectively. This is because during that time the mobile phones are trying to hook up to a nearby base station and subsequently reaching out to the desired base station of which is nearest to the call destination.
Mobile phones are generally found to emit lower electric field when they are in receiving and sending message mode as compared to when they are at incoming and outgoing call modes as shown in Fig. 4. The highest and lowest electric field with strength of 6.0 and $3.7 \mathrm{~V} / \mathrm{m}$ are emitted by mobile phones Brand SN1 and NK5, respectively. This low field strength is because when a message is successfully received or sent, the phone is in a one way transmitter or receiver condition. On the other hand, during the incoming and outgoing call modes the phone is having a wireless connection with another phone, which basically is a two way transmitter or receiver condition.

Basically, from Fig. 2-4, with a comparison to the ICNIRP guidelines for limiting exposure to general public, it is found that mobile phones Brand NK1, SN1 and SN2 emitted electric field to its surroundings at a higher level compared to the guideline. On the other hand, mobile phones Brand NK2, NK3, NK4, NK5, SM1, SM2, SG1, SG2 and MT1 emitted lower level electric field to its surroundings compared to the guidelines.

Hence, there is a need to ensure that while using the mobile phones to be connected to the world, mobile phone users are kept safe from such high electric field exposure. Not only will this help users from having light health problem such as dizziness, headache, it will also help the users to reduce the probability to have cancer in a long run.

\section{DISCUSSION}

Researches done around mobile phones were meant to determine the possible health effect and are inconclusive to date. While some researchers were able to find a link between mobile phones radiation and some negative health effect ${ }^{[5,7,10-12]}$, others are at the opposite ${ }^{[13,14]}$ more work can be found on WHO EMF project website. Based on our review there wasn't any research that investigate the electromagnetic field strength emitted by mobile phones at four different mode of operation while facing and backing the object. The findings of this research was contrary to the assumption of some researches and mobile phones operators that mobile phones electromagnetic field radiation emission are far below the widely used ICNIRP guidelines, this work has demonstrated that some mobile phones emitted higher electric field strength in active mode as compared to the guidelines. However, some of the mobile phones under test exhibit a lower emission of electric field strength as compared to similar guidelines. The electric field strength was found to depend on the mode of operation, the 
proximity of the phones to both the base station and the human body. From the results it is found that electric field strength emission was higher during outgoing call mode with the phone backing the measurement probe and lower when at SMS receiving mode. The high intensity electric field generated by some brand of mobile phones as shown in the results of this finding may cause tissue mutilation which may result to cancer. It is recommended that in carrying out research on possible effect of electromagnetic field around a mobile phones, intermittent long term exposure study putting into consideration the various modes of operation as shown in this study be considered.

\section{CONCLUSION}

Various guidelines exist for limiting exposure to radio frequency electromagnetic field by different countries. Where the most common one being the ICNIRP guidelines with $41.25 \mathrm{~V} / \mathrm{m}$ as the limit above which is not permitted to exceed at $900 \mathrm{MHz}$. Many countries guidelines are far below this due to complain and skepticism demonstrated by general public. The results presented in this work shows some mobile phones in compliance with the ICNIRP guidelines, some readings very close to the guidelines while others are far above the guidelines set by ICNIRP.

Mobile phones should be use at SMS mode and should be prevented from backing part of human body, as the radiation emission is lower at SMS mode and higher when backing the phones at outgoing call mode. This is necessary due to the growing state of uncertainty surrounding the bulk of evidences of harmful on one side and not harmful on the other side when using mobile phones.

\section{REFERENCES}

1. Hamadoun, T., 2008. Role of technological innovation in meeting MDGs. ITU Press Release.

2. Habash, R.W.Y., 2001. Electromagnetic Fields and Radiation. CRC Press, Marcel Dekker, New York, ISBN: 10: 0824706773, pp: 416.

3. International Nonionizing Radiation Committee of the International Radiation Protection Association, 1998. Guidelines for limiting exposure to timevarying electric, magnetic and electromagnetic fields (up to $300 \mathrm{GHz}$ ). Health Phys., 74: 494-522. http://www.ncbi.nlm.nih.gov/pubmed/9525427

4. Poljack, D., 2002. Electromagnetic Modeling of Wire Antenna Structures. WIT Press, Southampton, ISBN: 0890063605, pp: 408.
5. Silverman, C., 1980. Epidemiologic studies of microwave effects. Proc. IEEE., 68: 78-84. http://ieeexplore.ieee.org/xpl/freeabs_all.jsp?arnum ber $=1455854$

6. Robinette, C.D., C. Silverman and S. Jablon, 1980. Effect upon Health of occupational exposure to microwave radiation (radar) 1950-1974. Am. J. Epidemiol., $\quad 112$ : http://www.ncbi.nlm.nih.gov/pubmed/7395854

7. Hardel, L., A. Nasman, A. Pahlson, A. Hallquist and K.H. Mild, 1999. Use of cellular telephones and the risk for brain tumors: A case control studies. Int. J. Oncol., 15: 113-116. http://cat.inist.fr/?aModele $=$ afficheN\&cpsidt $=1861$ 840

8. Gandhi, O.P. and J.Y. Chen, 1992. Numerical dosimetry at power-line frequencies using anatomically based models. Bioelectromagnetics, 1: 43-60. http://www.ncbi.nlm.nih.gov/pubmed/1285721

9. Poljack, D. and V. Roje, 1998. Current induced in human body exposed to power line electromagnetic field. Proceedings of 20th Annual Conference IEEE Engineering Medical Biological Society, Oct. 29-Nov. 01, IEEE Xplore Press, Hong Kong, China, pp: 3281-3284. DOI: 10.1109/IEMBS.1998.746198

10. Lim, H.B., G.G. Cook, A.T. Baker and L.A. Coulton, 2002. FDTD design of RF dosimetery apparatus to quantify the effects of near fields from mobile handsets on stress response mechanisms of human whole blood. Int. J. Numer. Model. 15: 563-577. DOI: 10.1002/jnm.465

11. Muscat, J.E., M.G. Malkin, S. Thompson, R.E. Shore and S.D. Stelman et al., 2000. Handheld cellular telephone use and risk of brain cancer. J. Am. Med. Assoc., 284: 3001-3007. http://jama.amaassn.org/cgi/content/abstract/284/23/3001

12. Li, Yang., G, Lu, 2008. Discussion on biological effect of mobile phone radiation. Proceeding of the International Conference on Microwave and Millimeter Wave Technology, Apr. 211-24, IEEE Xplore Press, USA., pp: 1711-1713. DOI: 10.1109/ICMMT.2008.4540801

13. Rapacholi, M.H., J.A.J, Stolwijk, 1991. Cretaria for evaluating scientific literature and developing exposure limits. Radiat. Project Dosim., 9: 79-84.

14. Velberg, P.A., T.E. van Devanter and M.H. Repacholli, 2007. Workgroup report: Base stations and wireless networks-radiofrequency exposures and health consequences. Environ. Health Perspect., 115: 416-424. http://www.ncbi.nlm.nih.gov/pubmed/17431492 